\title{
Agronomic
} Journal Ipê

\section{VIABILIDADE ECONÔMICA DE IMPLANTAÇÃO HIDROPÔNICA NA CULTURA DA ALFACE}

\section{ECONOMIC VIABILITY OF HYDROPONIC IMPLANTATION IN ALFACE CULTURE}

Igor Leonardo Vespucci ${ }^{1}$, Daniel Soares Brito ${ }^{2}$; Milanna Paula Cabral Nunes ${ }^{3}$; Gustavo Henrique Mendes Brito 4

\footnotetext{
${ }^{1}$ Bacharel em Agronomia, doutorando em Agronegócio, Programa de Pós-Graduação em Agronegócio da Universidade Federal de Goiás (UFG), igorvespucci@agronomo.eng.br;

${ }^{2}$ Bacharel em Engenharia agronômica pela UniMB, dani3llbritts@hotmail.com;

${ }^{3}$ Bacharel em agronomia, mestrando em Engenharia agrícola pelo Programa de Pós-Graduação em Engenharia Agrícola da Universidade Estadual de Goiás (UEG), milannanunes@icloud.com;

${ }^{4}$ Docente, Mestre em Engenharia Agrícola da Faculdade Evangélica de Goianésia, gh.mendes.brito@gmail.com
}

\section{Info}

Recebido: 09/2019

Publicado: $11 / 2019$

ISSN: 2595-6906

Palavras-Chave: Lucro, Retorno, Tempo.

.Keywords: Profit, Return, Time.

\section{Resumo}

Em consequência do aumento populacional tem havido a necessidade de se produzir mais em um menor espaço observando os cuidados com o meio ambiente. Sendo assim, o homem vem procurando novas formas de cultivo como a hidroponia. O presente trabalho tem por objetivo avaliar a viabilidade econômica da implantação hidropônica na cultura da alface. Utilizou-se os seguintes métodos de análise de viabilidade: VPL, TIR e Pay back. Após a análise destas três

ferramentas, fica constatado que é economicamente viável a implantação de um projeto hidropônico na cultura da alface, no qual o investimento veio a apresentar um retorno financeiro em tempo menor que um ano, sendo capaz de produzir lucros a partir do primeiro ano de produção. Portanto, nota-se que trata-se de uma cultura totalmente viável economicamente para o cultivo em hidroponia.

\section{Resumo}

As a consequence of the population increase there has been a need to produce more in a smaller space observing the care with the environment. Thus, man has been looking for new forms of cultivation such as hydroponics. The present work aims to evaluate the economic viability of hydroponic implantation in lettuce culture. The following viability analysis methods were used: NPV, TIR and Pay back. After the analysis of these three tools, it is verified that it is economically feasible to implement a hydroponic project in the lettuce crop, in which the investment came to show a financial return in less time than a year, being able to produce profits from the first year of production. Therefore, it is noteworthy that this is a totally economically viable crop for hydroponic cultivation.

\section{INTRODUÇÃO}

De origem asiática e trazida para o Brasil pelos portugueses no século XVI, a alface (Lactuca sativa L.) faz parte da família Asteraceae. (FELTRIN et al., 2009). Santana et al., (2006) cita a alface como a folhosa mais consumida no Brasil. As variedades comerciais são classificadas em crespa, mimosa, americana, lisa e romana (GREGÓRIO et al., 2012). O comércio da variedade crespa corresponde a $60 \%$ e lisa com 19\% dentre as consumidas no país (CAETANO, 2001; BALINELO et al., 2009).

Como consequência do grande aumento populacional, e também da necessidade do consumidor mudar de hábitos alimentares, tem-se notado que esses fatores vêm gerando um grande aumento no consumo de hortaliças. De acordo com Potrich et al. (2012), a degradação do meio ambiente vem despertando uma grande preocupação quanto a sustentabilidade das 
atividades humanas, uma vez que, uma parte considerável do ecossistema está sendo alterada de forma devastadora pelo homem e a exploração desenfreada dos recursos naturais tem ultrapassado a capacidade de recuperação de vários ecossistemas.

Diante das alterações ambientais provocadas pelo ser humano, a hidroponia vem sendo considerada uma alternativa. Segundo Resh (1997) a hidroponia é uma forma alternativa de substituição do solo por elementos líquidos e que possuem apenas os nutrientes necessários para as plantas. Lopes et al. (2005) observa que umas das principais vantagens de cultivo através da hidroponia é a redução de riscos causados por patógenos de solo, que é uma das principais ameaças a produção de hortaliças

Nos dias de hoje, a alface pode ser considerada a hortaliça mais consumida no Brasil (HEREDIA ZÁRATE et al., 2010). A alface possui propriedades tranquilizantes com alto teor de vitaminas $\mathrm{A}, \mathrm{B}$ e $\mathrm{C}$ e ainda potássio, fósforo entre outros minerais. (VIGGIANO, 1990).

Rebellato (2004) ressalta que, objetivando a orientar profissional para análise de investimentos quanto à tomada de decisões financeiras, de maneira segura e rápida, faz se necessário analisar, de forma mais detalhada, os investimentos a serem aplicados na hidroponia. Não é recomendado que na elaboração de um projeto venha se optar por separar o ato de investir e financiar, já que, dificilmente, ambos serão independentes, porque o capital é um fator de produção e, na sua maioria, possui custo associado (BOLDA et al., 2011). O principal objetivo de se levantar o custo de produção é dar um parâmetro para a análise da rentabilidade (TAKAKI, 1999).

Sendo assim, verificando a necessidade de alta produção da alface no país, um dos maiores questionamentos que se faz é o que tange ao dispêndio econômico que se tem para implantar tal atividade. A análise desse questionamento é o objeto de pesquisa deste trabalho, permitindo verificar os efeitos positivos da hidroponia. Logo, o objetivo deste trabalho foi avaliar a viabilidade econômica da implantação hidropônica na cultura da alface.

\section{MATERIAL E MÉTODOS}

O presente trabalho foi realizado após um levantamento do custo de implantação de uma estufa hidropônica na cidade de Americano do Brasil-GO. Os dados foram fornecidos por uma empresa especializada em hidroponia situada nessa cidade. A estufa possui uma área total de 210 metros quadrados e capacidade para a produção estimada de 3 mil e 100 mudas. Para a análise de viabilidade foi construído um quadro com os custos de implantação do projeto (Quadro 1).

Quadro 1 - Custos de implantação da estufa hidropônica para produção de alface na cidade de Americano do Brasil-GO.

\begin{tabular}{|c|c|c|}
\hline Material Permanente & Quantidade & Valores \\
\hline Parte aérea de $7 \mathrm{~m} \times 30 \mathrm{~m}$ com sistema de molas incluso & 1 & $8.105,60$ \\
\hline Pés metálicos centrais de $7,00 \mathrm{~m}$ & 2 & 600,00 \\
\hline Pés metálicos laterais de $4,80 \mathrm{~m}$ & 22 & $2.706,00$ \\
\hline Sistema de cabeamento completo & 1 & $1.258,48$ \\
\hline $\begin{array}{l}\text { Filme Ginegar (Israel) difusor 52\% com } 5 \text { camadas, } 120 \text { micras, antiestático (reduz poeira) de } \\
\qquad 8,6 \mathrm{~m} \times 32 \mathrm{~m} \text { ou } 11 \mathrm{~m} \times 32 \mathrm{~m}(1 \text { rolo de } 5 \mathrm{~m} \text { e } 1 \text { rolo de } 6 \mathrm{~m})\end{array}$ & 1 & $1.383,36$ \\
\hline Tela lateral Polysombra preta $35 \%$ de $6,5 \mathrm{~m}$ x $100 \mathrm{~m}$ & 1 & $2.366,00$ \\
\hline Porta de correr, metálica, vedada com tela, de 1,10 x 2,20 m & 1 & 660,00 \\
\hline \multicolumn{2}{|l|}{ Custo Total (R\$) } & $17.079,44$ \\
\hline
\end{tabular}




\begin{tabular}{|c|c|c|}
\hline Perfis TP58 de 4,50 m c/ furo $10 \mathrm{~cm}$ (com bolsa) & 54 & 972,00 \\
\hline Perfis R80 de 4,50 m c/ furo $25 \mathrm{~cm}$ (com bolsa) & 126 & $3.855,60$ \\
\hline Tampões de entrada para perfil TP58 & 32 & 41,60 \\
\hline Perfil de recolhimento de $0,75 \mathrm{~m} \times 9$ encaixes TP58 & 3 & 63,00 \\
\hline Suportes para perfil TP58 (não acompanham parafusos) & 167 & 125,25 \\
\hline Tampões para perfil R80 & 68 & 129,20 \\
\hline Perfil de recolhimento de $1,45 \mathrm{~m} \times 7$ encaixes $\mathrm{R} 80$ & 9 & 365,40 \\
\hline Suportes para perfil R80 (não acompanham parafusos) & 257 & 231,30 \\
\hline Sistema injetor de solução completa & 95 & 228,00 \\
\hline Cano PP especial de $50 \mathrm{~mm} \times 6 \mathrm{~m}$ & 6 & 210,00 \\
\hline Cano PP especial de $25 \mathrm{~mm} \times 6 \mathrm{~m}$ & 6 & 120,00 \\
\hline Perfil de bancada - barra de $0,70 \mathrm{~m}$ (Travessa de cavalete) & 30 & 150,15 \\
\hline Perfil de bancada - barra de $1,40 \mathrm{~m}$ (Travessa de cavalete) & 63 & 630,63 \\
\hline Pés de bancada de 1,20 m (Pés de cavalete) & 186 & $1.595,88$ \\
\hline Selacalha acrílica & 3 & 48,90 \\
\hline \multicolumn{2}{|l|}{ Custo Total das Bancadas Hidropônicas (R\$) } & $8.766,91$ \\
\hline Painel elétrico c/ relê, timer, liga-desliga 220V & 1 & 700,00 \\
\hline $\begin{array}{l}\text { Bomba centrifuga 3/4 H.P monofásica THEBE (THB13) } \\
\text { Sucção: 2" / Recalque: 11ำ” / Vazão Máxima: } 19,1 \mathrm{~m}^{3} / \mathrm{h}\end{array}$ & 1 & $1.500,00$ \\
\hline Peagâmetro digital HM PH 80 & 1 & 220,00 \\
\hline Condutivímetro digital HM COM 80 & 1 & 199,00 \\
\hline Kit de soluções de calibração para aparelhos (pH 4, pH 7 e condutividade 1413) - $500 \mathrm{ml}$ & 1 & 175,00 \\
\hline Venturi 3/4” & 1 & 40,00 \\
\hline \multicolumn{2}{|l|}{ Custo Total dos Equipamentos (R\$) } & $2.834,00$ \\
\hline Solução nutritiva Hidrogood Fert- sc de $25 \mathrm{~kg}$ & 1 & 152,50 \\
\hline Nitrato de Cálcio sc de $25 \mathrm{~kg}$ & 1 & 61,50 \\
\hline Ferro EDDHA 6\% sc $1 \mathrm{~kg}$ & 1 & 60,00 \\
\hline Espuma fenólica Oasis (caixa c/ 10.350 células) & 1 & 133,00 \\
\hline Bandeja para espuma fenólica & 5 & 65,00 \\
\hline Custo total dos insumos $(\mathbf{R} \$)$ & & 472,00 \\
\hline
\end{tabular}


A fim de sintezir as informações fora construído a tabela 1.

Tabela 1 - Descrição dos custos finais para implantação de um sistema hidropônico.

\begin{tabular}{cc}
\hline Itens Necessários & Perfil PREMIUM dupla camada \\
\hline Bancadas hidropônicas & $8.766,91$ \\
\hline Equipamentos elétricos e Aparelhos & $2.834,00$ \\
Insumos & 472,00 \\
Reservatório 2.000 L para alface & 800,00 \\
Estufa & $17.079,44$ \\
Remuneração Eng. Agronômico (Anual) & $11.448,00$ \\
Total: & $\mathbf{4 1 . 4 0 0 , 3 5}$ \\
\hline
\end{tabular}

Para avaliar os aspectos financeiros da alface hidropônica, foram utilizados gráficos e tabelas que estimaram os custos e o lucro da atividade. As análises quantitativas referentes à decisão de investir foram feitas a partir das projeções do projeto, a fim de auxiliar na tomada de decisão de se investir ou não na inovação recém-elaborada. Todos os cálculos foram baseados nos estudos de Noronha (1987). Para o Pay back Simples (PBS), que corresponde ao período de tempo necessário para que o capital investido inicialmente no projeto seja recuperado, será utilizada a equação abaixo que será expressa por:

$$
P B S=\sum_{t=0}^{n}(B-I)_{t}=0
$$

Em que:

$B$, são os benefícios;

I, é o investimento no instante inicial;

t, o período de tempo;

n, é o horizonte do investimento.

O TIR é a taxa máxima de desconto que o investimento suporta e que iguala o VPL a zero. Caso a TIR seja maior que a taxa de desconto predeterminada, o empreendimento será economicamente viável.
A Taxa Interna de Retorno (TIR) é a taxa de desconto interna gerada pelo investimento que torna o $\mathrm{VPL}=0$, apresentado pela equação abaixo:

$$
V P L=\sum_{t=1}^{n} \frac{(R-C)_{t}}{(1+T I R)}=0
$$

Em que:

$\mathrm{R}$, são as receitas;

C, os custos e os investimentos gerados;

t, o período de tempo;

$\mathrm{n}$, o horizonte do investimento.

O Valor Presente Líquido (VPL) representa o retorno monetário do investimento, considerando o valor do dinheiro no tempo, a uma taxa de desconto predeterminada, equação abaixo:

$$
V P L=\sum_{t=1}^{n} \frac{(R-C)_{t}}{(1+k)^{t}}
$$

Em que:

$\mathrm{R}$, são as receitas;

C, os custos e os investimentos gerados pelo projeto;

t, o período de tempo;

$\mathrm{n}$, o horizonte do investimento;

$\mathrm{k}$, a taxa de desconto utilizada. 
Pelo critério considerado, quando VPL $>0$, o investimento é viável; quando $\mathrm{VPL}<0$, o investimento é inviável e VPL $=0$, indiferente.

\section{RESULTADOS E DISCUSSÃO}

Os resultados obtidos tiveram como base os valores levantados para implantação do projeto (Quadro 1; Tabela 1) os quais têm como função a projeção estipulada de produção de alface, a partir do resultado do custo de implantação (Tabela 2).

Nota-se que a TIR a um taxa de desconto de $20 \%$ obteve o valor de $135,05 \%$, segundo Noronha
(1987) quando a TIR é maior que a taxa de desconto predeterminada, o empreendimento será economicamente viável. Rezende e Oliveira (2001) ainda afirmam que, no caso do projeto apresentar um VPL > 0, pode-se tê-lo como viável, porque mostra que o valor do investimento hoje é maior do que o valor do investimento de início, levando em consideração o custo de investimento do produtor. Neste caso, o VPL possui um valor substancialmente maior que 0 mostrando de forma segura que este projeto é um investimento viável.

Tabela 2 - Calculo de VPL, TIR, e Pay Back com uma taxa de desconto (i) de 20\% calculado no programa Excel para produção de alface hidropônica.

\begin{tabular}{|ccc}
\hline \multicolumn{1}{c}{ Ano } & Projeto \\
\hline 0 & $\mathrm{R} \$$ & $-41.400,35$ \\
1 & $\mathrm{R} \$$ & $56.700,00$ \\
2 & $\mathrm{R} \$$ & $56.700,00$ \\
3 & $\mathrm{R} \$$ & $56.700,00$ \\
4 & $\mathrm{R} \$$ & $56.700,00$ \\
5 & $\mathrm{R} \$$ & $56.700,00$ \\
\hline (i) & & $20 \%$ \\
\hline TIR & \multicolumn{2}{c}{$135,05 \%$} \\
VPL & $\mathrm{R} \$$ & $128.167,36$ \\
Pay back & \multicolumn{2}{c}{0,73} \\
\hline
\end{tabular}

O tempo estipulado para o retorno do investimento foi de 5 anos e o Pay back mostrou que o projeto é capaz de trazer resultados em um tempo menor que 1 ano (Figura 1). Nesta análise, a unidade da alface foi cotada a $R \$ 1,50$. Ao avaliar a produção em outras regiões pode haver alteração no valor unitário, fazendo com que o projeto se torne ainda mais viável que $\mathrm{o}$ analisado. 


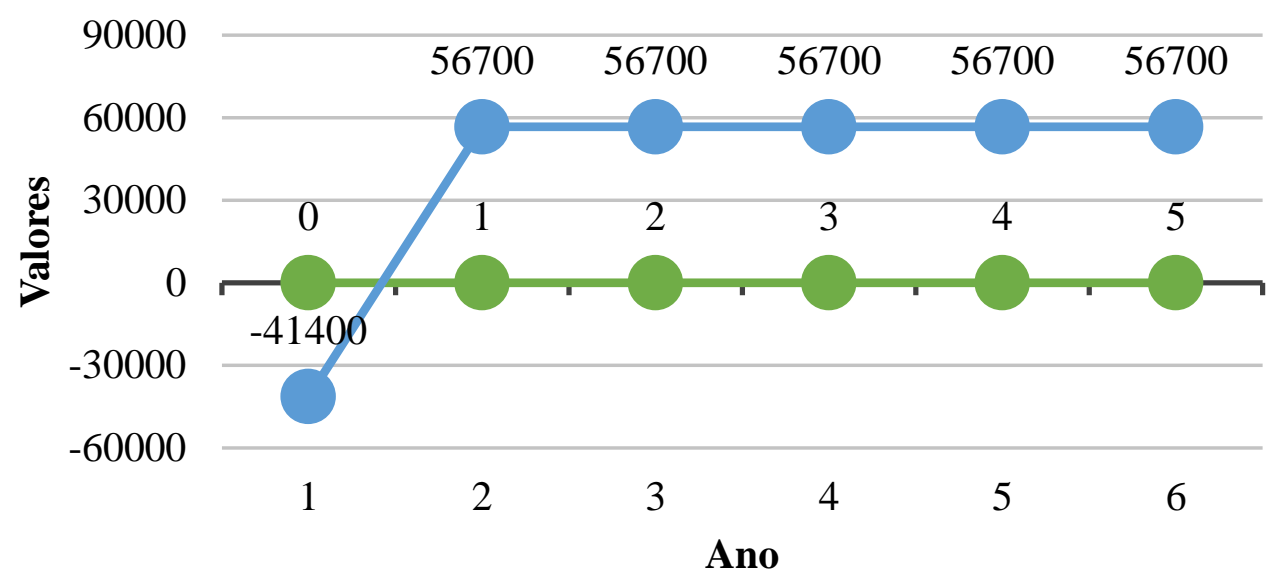

Figura 1 - Tempo de retorno da análise de viabilidade econômica de implantação da alface hidropônica

A figura 1 se refere ao resultado final da viabilidade econômica de implantação de alface hidropônica, tal retorno se inicia antes de um ano de implantação. Dal'Sotto (2013) relata que a hidroponia é um investimento viável na agricultura familiar, com um retorno de investimento consideravelmente rápido do investimento e com uma boa renda, representando uma forma eficaz de investimento no meio rural. Contudo, contrariando tais resultados, Geisenhoff et al. (2009) verifica que a atividade se encontra em processo de descapitalização, a receita total é suficiente apenas para cobrir as despesas de custos variáveis e parte dos custos fixos, não sendo possível cobrir os custos totais.

\section{CONCLUSÃO}

Considerando que com o aumento populacional nota-se uma necessidade de se produzir cada vais mais uma maior quantidade de alimentos em um espaço cada vez menores, a hidroponia é uma forma viável de se produzir, já que ela necessita de uma área relativamente menor comparada ao cultivo convencional. Portanto, conclui-se que na região analisada o cultivo hidropônico de alface mostra-se economicamente viável, apresentando um resultado positivo. O tempo de pagamento dos custos de implantação foi menor que 1 ano e gerando renda ao agricultor após este período.

\section{REFERÊNCIAS}

BALINELO, V.J.; GOUVÊIA, M.I.D.; COELHO, M.P.; ZAMPROGNO A.C.; FIANCO, B.A.; OLIVEIRA, L.G.A. Enteroparasitas em hortaliças comercializadas na cidade de São Mateus, ES, Brasil. Arquivo Ciência SaúdeUNIPAR, Umuarama, v. 13, n. 1, p. 33-36, 2009. Disponível em: < http://revistas.unipar.br/index.php/saude/artic le/view/2794>. Acesso em: 15 de fev. 2019.

BOLDA. M.; LAURA, T.; KAREN, M.; KLONSKY; RICHARD, L.M. Sample Costs to Produce Second Year Strawberries. Davis: University of California Cooperative Extension, 2011.

CAETANO, L.C.S. A cultura da alface: perspectivas, tecnologias e viabilidade. Niterói: Varela, 2001. Disponível em:< http://www.bc.ufra.edu.br/handler.php?modul $\underline{\mathrm{e}=\text { gnuteca\&action }=\text { main:pesquisa:lista\&frm ex }}$ $\underline{\text { ato }}=1 \&$ frm etiqueta $=245 . \mathrm{a} \&$ frm conteudo $=\mathrm{A}$ + cultura + da + alface $\% 3 \mathrm{~B}+$ perspectivastecnologias $+\mathrm{e}+$ viabilidade $\&$ frm unidade $=1>$. Acesso em: 06 de dez 2018.

DAL'SOTTO, T.C. Estudo de viabilidade econômica para implantação de um sistema de cultivo hidropônico em uma propriedade rural no oeste do Paraná. 2013. 58-60 f. Trabalho de conclusão de curso (Graduando em Engenharia Agronômica) - Universidade Tecnológica Federal do Paraná, Paraná, 2013. Disponível em: < http://repositorio.roca.utfpr.edu.br/jspui/hand le/1/1663 >. Acesso em: 22 de jan. 2019. 
FELTRIN, A.L.; CECILIO FILHO, A.B.; REZENDE, B.L.A.; BRANCO, R.B.F.

Produção de alface americana em solo e em hidroponia,no inverno e verão,em Jaboticabal, SP. Científica, Jaboticabal, v.37, n.1, p.9-15, 2009. Disponível em: <

http://www.scielo.br/scielo.php?pid=S141543662005000400010\&script $=$ sci abstract\&tlng $\equiv \mathrm{pt}>$. Acesso em: 20 de abril. 2019.

GEISENHOFF, L. O.; PEREIRA, G.M.; FARIA, L.C.; JUNIOR, J.A.L.; COSTA, G.G.; GATTO, R.F. Viabilidade econômica da produção de alface hidropônica em Lavras-MG. Agrarian, v.2, n.6, p.61-69, 2009. Disponível em: < http://repositorio.roca.utfpr.edu.br/jspui/hand le/1/1663 >. Acesso em: 22 de jan. 2019.

GREGÓRIO, D.S.; MORAES, G.F.A.; NASSIF, J.M.; ALVES, M.R.M.; CARMO, M.E.;

JARROUGE, M.G.; BOUÇAS, R.I.; SANTOS, A.C.C.; BOUÇAS, T.R.J. Estudo da contaminação por parasitas em hortaliças da região leste de São Paulo. Revista Science in Health, v. 3, n. 2, p. 96-103, 2012. Disponível em: $<$

http://ojs.ufgd.edu.br/index.php/agrarian/arti cle/view/931 >. Acesso em: 22 de jan. 2019.

HEREDIA ZÁRATE, N.A.; VIEIRA, M.C.; HELMICH, M.; HEID, D.M; MENEGATI, C.T. Produção agroeconômica de três variedades de alface: cultivo com e sem amontoa. Revista Ciência Agronômica, Iguatu, v. 41, n. 4, p. 646-653, 2010. Disponível em: $<$

http://ccarevista.ufc.br/seer/index.php/ccarevi sta/article/view/996/0>. Acesso em: 02 de nov. 2018.

LOPES, C.A.; CARRIJO, O.A.; MAKASHIMA, N. Contaminação com patógenos em sistemas hidropônicos: como aparecem e como evitar. 31. ed. Brasilia: Embrapa, 2005.

NORONHA, J.F. Projetos agropecuários; administração financeira, orçamento e viabilidade econômica. São Paulo: Editora Atlas, 1987.

POTRICH, A.C.; PINHEIRO, R.R.; SCHMIDT, D. Alface Hidropônica como alternativa de produção de alimentos de forma sustentável. Enciclopédia Biosfera, Goiânia, v.8, n.15, p.36, 2012. Disponível em: <http://www.conhecer.org.br/enciclop/2012b /ciencias\%20agrarias/alface.pdf $>$. Acesso em: 22 de jan. 2019.

RESH. H.M. Cultivos hidropônicos: nuevas técnicas de producción. 4 ed. Madrid: Ediciones Mundi-Prensa, 1997.

REZENDE, J.L.P.; OLIVEIRA, A.D. Análise econômica e social de projetos

florestais. Viçosa: Editora UFV, 2001.

SANTANA, L.R.R.; CARVALHO, R.D.; LEITE, C.C.; ALCÂNTARA, L.M.; OLIVEIRA, T.W.S.; RODRIGUES, B.D.M. Qualidade física, microbiológica e parasitológica de alfaces. Food Science Technology, Campinas, v. 26, n. 1, p. 264-269, 2006. Disponível em: < http:/ / www.scielo.br/scielo.php?pid=S0101$20612006000200006 \&$ script $=$ sci abstract $\&$ th Ept>. Acesso em: 12 de jun. 2019.

TAKAKI, H.R.C. Introdução à economia para micro e pequenas empresas. Lavras: 1999.

VIGGIANO, J. Produção de sementes de alface. In: CASTELLANE, P.D.; NICOLOSI, W.M.; HASEGAWA, M. Produção de sementes hortaliças. Jaboticabal: FCAV/FUNEP, 1990. p. 1-13. 